\title{
Fast SRC using Quadratic Optimisation in Downsized Coefficient Solution Subspace
}

\author{
Xiaoning Song ${ }^{\mathrm{a}, \mathrm{b}, *}$, Guosheng $\mathrm{Hu}^{\mathrm{c}}$, Jian-Hao Luo ${ }^{\mathrm{d}}$, Zhenhua Feng ${ }^{\mathrm{c}}$, Dong-Jun \\ $\mathrm{Yu}^{\mathrm{e}}$, Xiao-Jun $\mathrm{Wu}^{\mathrm{a}, \mathrm{b}}$ \\ ${ }^{a}$ School of IoT Engineering, Jiangnan University, Wuxi, China \\ ${ }^{b}$ Jiangsu Provincial Engineering Laboratory of Pattern Recognition and Computational \\ Intelligence, Jiangnan University, Wuxi, China \\ ${ }^{c}$ Center for Vision, Speech and Signal Processing, University of Surrey, Guildford, UK \\ ${ }^{d}$ National Key Laboratory for Novel Software Technology, Nanjing University, Nanjing, \\ China \\ ${ }^{e}$ School of Computer Science and Engineering, Nanjing University of Science and \\ Technology, Nanjing, China
}

\begin{abstract}
Extended sparse representation-based classification (ESRC) has shown interesting results on the problem of under-sampled face recognition by generating an auxiliary intraclass variant dictionary for the representation of possible appearance variations. However, the method has high computational complexity due to the $l_{1}$-minimization problem. To address this issue, this paper proposes two strategies to speed up SRC using quadratic optimisation in downsized coefficient solution subspace. The first one, namely Fast SRC using Quadratic Optimisation (FSRC-QO), applies PCA and LDA hybrid constrained optimisation method to achieve compressed linear representations of test samples. By design, more accurate and discriminative reconstruction of a test sample can be achieved for face classification, using the downsized coefficient space. Secondly, to explore the positive impact of our proposed method on deep-learning-based face classification, we enhance FSRC-QO using CNN-based features (FSRC-QO$\mathrm{CNN}$ ), in which we replace the original input image using robust CNN features in our FSRC-QO framework. Experimental results conducted on a set of wellknown face datasets, including AR, FERET, LFW and FRGC, demonstrate the
\end{abstract}

\footnotetext{
* Corresponding author

Email address: x.song@jiangnan.edu.cn (Xiaoning Song)
} 
merits of the proposed methods, especially in computational efficiency.

Keywords: Fast SRC, dimensionality reduction, CNN-based features, face recognition.

\section{Introduction}

Face recognition (FR) is one of the most active topics in pattern recognition, computer vision and biometrics due to its wide range of applications such as access control, video surveillance, social network, photo management and criminal investigation, etc [1]. However, FR performance is often influenced by many factors including of poses, expressions, illuminations, ages, disguises and cluttered background. In fact, not all the measured observations with high dimensionality are important for the classification. Hence researchers have been making attempts to the invention of numerous methods for dimensionality reduction that can be used as one of the critical techniques for efficient face classification, including Principal Component Analysis (PCA) 2, Linear Discriminant Analysis (LDA) 3], Laplacian Eigenmaps [4, Locality Preserving Projections (LPP) [5] and Locally Linear Embedding (LLE) [6], etc. Among these typical feature transformation methods, PCA is an unsupervised method that projects high dimensional data into a lower dimensional space by seeking the direction of maximum variance for optimal data reconstruction. LDA is a supervised dimensionality reduction method which enables the samples in the new space to have the maximum ratio of the between-class distance to the within-class distance. The common aim of the above transformation methods is to transform samples into a new space where some important properties might hold [7.

As another popular concept, the success of compact representation in image processing triggers a great deal of effort on sparse representation. In particular, sparse representation-based classification (SRC) 8, 9] [10] 11] has drawn extensive attention due to its successful applications in image classification. The aim of SRC is to represent a new observation, also known as a signal or a sample, using a minimal number of training samples selected from an existing dictionary 
that consists of a number of observations across different classes. To achieve this objective, $l_{1}$-norm constraint is used as a regularization term in SRC to obtain sparse reconstruction coefficient vectors. However, in some practical scenarios such as CCTV security systems, only a few or even just a single image of a subject is available for training, which leads to a low quality dictionary that is insufficient for SRC. This is the well-known small-sample-size (SSS) problem [12. To alleviate the issue, some dictionary learning methods have been developed to enhance the performance of SRC. For example, Deng et al. extended SRC to perform under-sampled face recognition using an intraclass variant dictionary [13. Yang et al. and Zhu et al. 14, 15] introduced similarity and distinctiveness of features to present a more general model of collaborative representation based classification (CRC). Xu et al. [16] proposed an efficient SRC via improved norm minimization. Song et al. [17] proposed a half-face dictionary integration algorithm for representation-based classification, the strength of this method is that it is able to successfully construct the dual-column (row) half-face training matrix, while quantifying the integrated learning atoms that exert influence on signal reconstruction. The use of virtual face images [18, 19] has also been proven beneficial to a number of face analysis tasks such as face 45 recognition [20, 21] and facial landmark detection [22, 23, 24, 25, 26]. Facial symmetry property has also been widely used to quickly locate the candidate samples in face detection, alignment and classification [27, 28, 29]. In addition, Zhou et al. 30] proposed a global context verification scheme to filter false matches for copy detection. Xia et al. 31. proposed a privacy-preserving and copy-deterrence CBIR scheme using encryption and watermarking techniques. Li et al. 32 proposed a scheme to detect the copy-move forgery in an image, mainly by extracting the keypoints for comparison.

Although extensive studies on SRC in terms of sparse classification capacity, fewer attentions have been paid on the optimisation problem in downsized 55 coefficient solution subspace, for the purpose of less computational complexity. In this paper, we proposed two strategies: FSRC-QO and FSRC-QO-CNN. The former adopted PCA and LDA to speed up the traditional SRC with the idea of 
dimensionality reduction. The latter is designed to improve the accuracy which is inspired by the excellent discrimination of CNN-based features. It should be noted that FSRC-QO-CNN is based on the well trained feature maps obtained by our first strategy, and the training processing can be completed using offline mode. To this end, we use the pre-trained VGG16 model for feature extraction, then the aforementioned FSRC-QO method can be applied to the extracted CNN features for face classification. Therefore, the FSRC-QO-CNN method is actually an extension of the first one and it is intended to improve the accuracy.

The contributions of our work are threefold:

- We construct a quadratic optimisation combined with dimensionality reduction constraint which can achieve the more accurate reconstructed PCA coefficient of each class in both raw image space and principle coefficient space.

- We employ LDA to further enhance the discriminative capacity of the proposed FSRC-QO method. More specifically, the reconstucted PCA coefficients of training samples achieved by the PCA constrained optimisation model are projected to a LDA space for robust discriminant analysis.

- We also apply state-of-the-art deep CNN features to our FSRC-QO for improving the accuracy of the proposed system. To this end, we use the pre-trained VGG16 model for feature extraction. Then the aforementioned FSRC-QO method can be applied to the extracted CNN features for face classification.

80

The rest of the paper is organized as follows: Section 2 overviews the background of ESRC 13. It is the prerequisites to our method proposed in Section 3. Section 4 presents a theoretical analysis to the proposed method and Section 5 reports the results of comprehensive experiments conducted on the well-known AR, FERET, LFW and FRGC face datasets. Lastly, we summarize the paper in Section 6. 


\section{Review of ESRC}

Given the training samples of all $K$ classes as the matrix $\mathbf{A}=\left[\mathbf{A}_{1}, \mathbf{A}_{2}, \ldots, \mathbf{A}_{K}\right] \in$ $\Re^{d \times n}$, where $n$ is the total number of training samples and $d$ is the dimensionality of a sample. The main idea of ESRC is that the large deviation from the test image to the correct gallery images may be linearly approximated by the intraclass differences of generic subjects. To this end, we introduce an additional basis matrix $\mathbf{D}_{\mathbf{I}} \in \Re^{d \times p}$ (the dictionary size $p$ depends on the data source and construction method) [13 to represent the universal intraclass variant bases which can be acquired in various ways as long as they can reflect the intraclass difference. Then the linear representation of a testing sample $\mathbf{y} \in \Re^{d}$ can be rewritten as:

$$
\mathbf{y}=\mathbf{A} \boldsymbol{\alpha}+\mathbf{D}_{\mathbf{I}} \boldsymbol{\beta}+t
$$

where the intraclass variant matrix $\mathbf{D}_{\mathbf{I}}$ usually represents the significant variations including unbalanced illuminations, exaggerated expressions, or occlusions that cannot be modeled by the small dense noise $t$. Once there are redundant and overcomplete facial variant bases in $\mathbf{D}_{\mathbf{I}}$, the combination coefficients in $\boldsymbol{\beta}$ are naturally sparse. Hence, the sparse representation $\boldsymbol{\alpha}$ and $\boldsymbol{\beta}$ can be recovered simultaneously by solving the $l_{1}$-norm minimization.

$$
\left[\begin{array}{l}
\widehat{\boldsymbol{\alpha}}_{1} \\
\widehat{\boldsymbol{\beta}}_{1}
\end{array}\right]=\arg \min \left\|\left[\begin{array}{l}
\boldsymbol{\alpha} \\
\boldsymbol{\beta}
\end{array}\right]\right\|_{1}, \text { s.t. }\left\|\left[\widehat{\mathbf{A}}, \mathbf{D}_{\mathbf{I}}\right]\left[\begin{array}{l}
\boldsymbol{\alpha} \\
\boldsymbol{\beta}
\end{array}\right]-\mathbf{y}\right\|_{2} \leq \varepsilon
$$

where $\boldsymbol{\alpha}, \widehat{\boldsymbol{\alpha}}_{1} \in \Re^{n}, \boldsymbol{\beta}, \widehat{\boldsymbol{\beta}}_{1} \in \Re^{p}$ and $\varepsilon>0$ stands for an error tolerance. We can measure the propensity of the $k$ th class to represent the test sample after obtained the coefficient vectors. Hence the test sample reconstruction error for the $k$ th class is achieved as well as the intraclass variant bases can be calculated as below:

$$
r_{k}(\mathbf{y})=\left\|\mathbf{y}-\left[\mathbf{A}, \mathbf{D}_{\mathbf{I}}\right]\left[\begin{array}{c}
\delta_{k}\left(\widehat{\mathbf{x}}_{1}\right) \\
\widehat{\boldsymbol{\beta}}_{1}
\end{array}\right]\right\|_{2}
$$

where $\delta_{k}\left(\widehat{\mathbf{x}}_{1}\right)$ is a new vector whose only nonzero entries are the entries in $\widehat{\mathbf{x}}_{1}$ those are associated with class $k$, and the label of the test sample $\mathbf{y}$ is determined 
by

$$
\operatorname{Lable}(\mathbf{y})=\arg \min _{k}\left\{r_{k}(\mathbf{y})\right\}
$$

\section{The proposed method}

As discussed in Section 1, the problem of existing relevant algorithms for sparse representation-based classification is that they build on the intrinsic properties of a dataset, and they are unable to cater for a specific technology for evaluating the information redundancy between the gallery and query sets. The traditional optimisation problem of the above methods was solved by standard linear programming methods using original high-dimensional samples for the optimisation coefficient. In order to decrease the adverse effects caused by raw feature space, we present two schemes to identify representative samples with the best computational capacity to represent a new sample.

\subsection{FSRC-QO}

The objective of the proposed FSRC-QO is to jointly employ PCA and LDA hybrid constrained model to enhance the discriminatory capacity of SRC. That is, the first phase of the proposed method seeks to achieve a compressive linear representation of the test sample using the PCA constrained optimisation model. By design, the more accurate reconstruction of the test sample using both sample space and principle coefficient space can be achieved. The second phase further improves the discriminative capability of the PCA coefficient in representing a test sample. It thereby obtains a more competitive optimization model for face classification.

\subsubsection{The first phase of the FSRC-QO}

Assuming that there is a dataset with multiple images per subject, the samples of subject $k$ are stacked as vectors. Hence an intralass variant dictionary $\mathbf{D}_{\mathbf{I}}$ can be constructed by subtracting the natural image from other images of the same class for the purpose of training data augmentation. To improve the optimisation efficiency, we project the training samples into the PCA space in 
which a new sparse representation model with PCA constrained optimisation is designed as follows:

$$
J_{(\boldsymbol{z}, \boldsymbol{\beta})}=\arg \min _{(\boldsymbol{z}, \boldsymbol{\beta})}\left\{\left\|\mathbf{y}-\mathbf{U z}-\mathbf{D}_{\mathbf{I}} \boldsymbol{\beta}\right\|_{2}^{2}+\lambda_{1}\|\mathbf{z}\|_{2}+\lambda_{2}\|\boldsymbol{\beta}\|_{1}\right\}
$$

where $\mathbf{U}$ stands for the basis of PCA in terms of training samples, $\lambda_{1}$ and $\lambda_{2}$ are the small positive constants, $t \in \Re^{d}$ is a noise term with bounded energy $\|t\|_{2}<\varepsilon$, and the optimal error tolerance $\varepsilon>0$. The optimisation of Eq. (5) can be viewed as a typical least-square problem and $\boldsymbol{\beta}$ is obtained by

$$
\boldsymbol{\beta}=\left(\mathbf{D}_{\mathbf{I}}^{T} \mathbf{D}_{\mathbf{I}}+\lambda I\right)^{-1} \mathbf{D}_{\mathbf{I}}^{T}(\mathbf{y}-\mathbf{U} \mathbf{z})
$$

where $\lambda$ is a small positive constant and $I$ is the identity matrix. We can calculate $\mathbf{z}$ after obtained the coefficient vector $\boldsymbol{\beta}$ by

$$
\mathbf{z}=\mathbf{U}^{T}\left(\mathbf{y}-\mathbf{D}_{\mathbf{I}} \boldsymbol{\beta}\right)
$$

The coefficient vectors $\mathbf{z}$ and $\boldsymbol{\beta}$ using $l_{2}$-norm minimization can be iteratively computed by Eq.(6) and Eq.(7) until the predefined termination condition is satisfied.

Moreover, considering the sparsity of $\boldsymbol{\beta}$ especially in the case of complex practical scenarios such as the differences of poses, expressions, illuminations, disguises and cluttered background, we can find a more accurate measurement of $\boldsymbol{\beta}$ by the solution of $l_{1}$-norm minimization instead of Eq. (6) as below:

$$
\hat{\boldsymbol{\beta}}=\arg \min _{\boldsymbol{\beta}}\|\boldsymbol{\beta}\|_{1}, \text { s.t. }\left\|\mathbf{y}-\mathbf{U z}-\mathbf{D}_{\mathbf{I}} \boldsymbol{\beta}\right\|_{2} \leq \varepsilon
$$

Thus, the more accurate PCA coefficient $\mathbf{z}$ of the test sample can also be achieved by

$$
\mathbf{z}=\mathbf{U}^{T}\left(\mathbf{y}-\mathbf{D}_{\mathbf{I}} \hat{\boldsymbol{\beta}}\right)
$$

\subsubsection{The second phase of the FSRC-QO}

Providing that the coefficient matrix $\widehat{\mathbf{A}}$ in terms of training samples are obtained by Eq.(10).

$$
\widehat{\mathbf{A}}=\mathbf{U}^{T} \mathbf{A}
$$


We then use $\mathbf{W}$ which denotes the basis of LDA in terms of $\widehat{\mathbf{A}}$ to calculate the LDA projected test vector $\widehat{\mathbf{z}}$ and the projected training matrix $\widehat{\mathbf{A}}_{L D A}$, as below:

$$
\widehat{\mathbf{z}}=\mathbf{W}^{T} \mathbf{z}
$$

$$
\widehat{\mathbf{A}}_{L D A}=\mathbf{W}^{T} \widehat{\mathbf{A}}
$$

Meanwhile, each LDA coefficient of training classes can also be obtained by:

$$
\operatorname{centers}_{k}=\frac{1}{n_{k}} \sum_{j=1}^{n_{k}} \widehat{\widehat{\mathbf{x}}}_{k}^{j}
$$

where $k=1,2, \ldots K, K$ is the number of classes, $n_{k}$ stands for the number of

115 samples of the $k$ th class, and $\widehat{\widehat{\mathbf{x}}}_{k}^{j}$ denotes the coefficient of $j$ th sample from the $k$ th class in $\widehat{\mathbf{A}}_{L D A}$.

Lastly, we can measure the reconstruction error using the distance between the reconstructed LDA coefficient of the test sample and the reconstructed average coefficient for the $k$ th training class, as follows:

$$
D_{k}(\widehat{\mathbf{z}})=\left\|\widehat{\mathbf{z}}-\operatorname{centers}_{k}\right\|_{2}
$$

Hence, the label of the test sample is estimated using the index of the smallest values of the deviations over all $D_{k}(\widehat{\mathbf{z}}), k=1,2, \ldots, K$.

$$
\operatorname{Lable}(\mathbf{y})=\arg \min _{k}\left\{D_{k}(\widehat{\mathbf{z}})\right\}
$$

\subsection{FSRC-QO-CNN}

More recently, Convolutional Neural Networks (CNN) have been successfully used in a wide range of computer vision and pattern recognition applications and become the mainstream in face biometrics. A CNN network trained on a large number of face images is able to extract robust textural features for face recognition, across a variety of appearance variations such as pose, expression, illumination and occlusion. To further improve the accuracy of the proposed system, we also use CNN features in our FSRC-QO. Here, FSRC-QO performs 
125 used nearest neighbour classifiers with cosine distance and Euclidean distance. To this end, we use the pre-trained VGG-FACE model for feature extraction 33 . Then the aforementioned FSRC-QO method can be applied to the extracted CNN features for face classification. We use the term 'FSRC-QO-CNN' for this approach. The only difference between these two methods is that FSRC-QO uses the raw pixel intensities for face classification whereas FSRC-QO-CNN uses extracted CNN features from an original input face image.

\subsection{The pipeline of our algorithm}

The pipeline of the proposed algorithm is described as follows:

1:Input: Training samples $\mathbf{X}$ with $\mathrm{K}$ class, an intraclass variant dictionary D and test sample $\mathbf{y}$;

2:Output: The label of test sample;

3: Projecting the training samples into the PCA space and get the basis of PCA :U.

4: For $\mathrm{i}=1$ to $\mathrm{N}$ (the number of samples) calculating the coefficient by Eq.(7).

\section{5:end for}

6:Obtaining the solution of $l_{1}$-norm minimization by Eq. (8).

7: Get the PCA coefficient $\mathbf{z}$ of the test sample by Eq.(9)

8:Apply the LDA algorithm to calculate the projected vectors by Eq.(11) and Eq.(12)

9:Obtain the LDA coefficient of each training classes.

10: Compute the reconstructed error between each class and the test sample. 


\section{Analysis of the proposed method}

To reveal the nature of the proposed methods, in this section, we further analyse our FSRC-QO and FSRC-QO-CNN methods in terms of the rationales, correlations, potential advantages and flaws. In fact, the traditional optimisation problem of SRC was solved by standard linear programming methods using original high-dimensional samples for the optimisation coefficient, which typically ignore the information redundancy between the gallery and query sets and may lead to uncertainty in decision making. Motivated by this, in the present study, we are to develop two strategies for SRC to improve speed and performance as well.

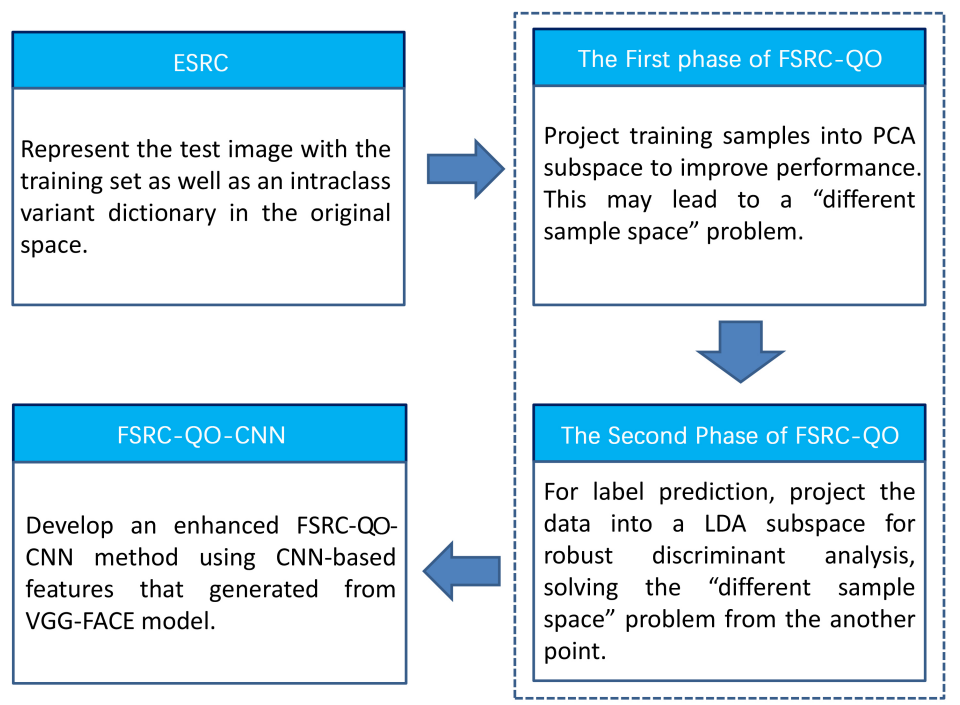

Figure 1: The correlations among the proposed methods.

\subsection{The correlations among the proposed methods}

As described in Section 3, two fast SRC algorithms are developed for the task of face classification. The strength of these strategies lies in successfully constructing some optimisation solutions using quadratic optimisation in downsized coefficient subspace, and thereby enhancing the collaborative and discriminative capacity of the dictionary to reconstruct the input images. FSRC-QO 
and FSRC-QO-CNN are both extended from ESRC, it is worth describing the correlations among the three algorithms, which is illustrated in the Fig. 1 .

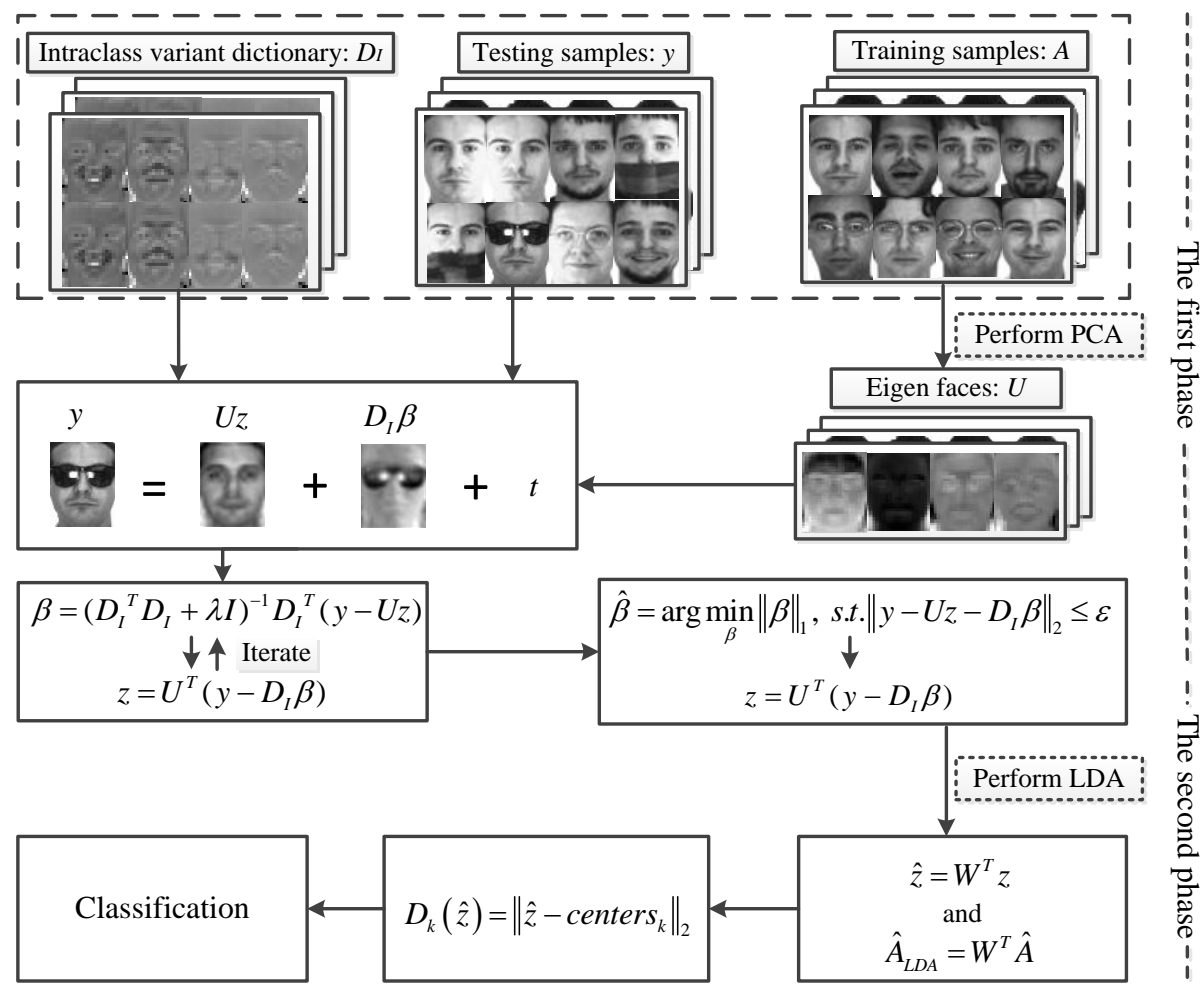

Figure 2: The schematic diagram of FSRC-QO.

To better illustrate how the proposed FSRC-QO works, we first provide a schematic diagram of FSRC-QO as Fig. 2. As shown in Fig. 2, The strategy works as an enhancement mechanism of discriminatory that applies PCA and LDA constrained model in two sequential sparse representation stages: the first 175 stage is to achieve the reconstructed PCA coefficient matrix $\widehat{\mathbf{A}}$ from the training samples, the second phase is to implement discriminant analysis to further maximize the ratio of between-class scatter coefficient matrix to that of within-class scatter coefficient matrix. 
To alleviate the adverse effect of the small-sample-size (SSS) problem, most

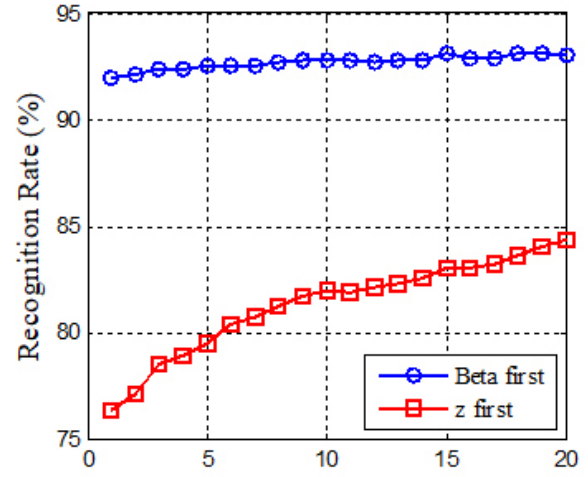

(a)

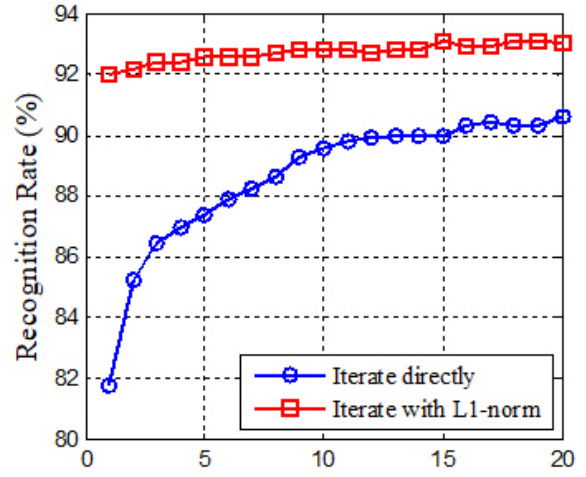

(b)

Figure 3: (a) The comparison results with different calculation sequence of coefficients. (b) The comparison results with different solution of optimisation problem on AR. 
by Eq.(6) and Eq.(7) until reach the predefined condition. In fact, the calculation sequence of the coefficient vectors $\boldsymbol{\beta}$ and $\mathbf{z}$ can affect the accuracy of the optimisation model. We are now making an analysis about the solving process of the optimisation problem as below. It is known that the coefficient vector $\mathbf{z}$ of FSRC-QO is obtained after solving $\boldsymbol{\beta}$, hence an interesting question arises: How is the performance of the optimisation problem while the calculation order of $\mathbf{z}$ is prior to $\boldsymbol{\beta}$ ? To illustrate this, Fig. 3(a) shows the comparison results with different calculation sequence of coefficients. As shown in Fig. 3(a), we can see that the recognition rate by solving $\boldsymbol{\beta}$ firstly is always higher than that of the opposite case as the the number of iteration increases. It is noted that the curve of the recognition rate with the first calculation of $\boldsymbol{\beta}$ become flat when the iteration number is increased to a certain extent (e.g.,>15), which implies that the excessive iterations between $\mathbf{z}$ and $\boldsymbol{\beta}$ might lead to over-fitting and timeconsuming. Thereby these findings motivate us to propose the PCA constrained optimisation model for the efficient classification.

After gaining the first solution of $\boldsymbol{\beta}$ using the typical least-square method as shown in Eq.(6), the first analytic solution $\mathbf{z}$ can be simultaneously obtained by Eq.(7). In fact, due to the sparsity of $\boldsymbol{\beta}$ especially in the case of complex practical scenarios, we can find a more accurate measurement of $\boldsymbol{\beta}$ by means of $l_{1}$-norm minimization as shown in Eq.(8). With this $\boldsymbol{\beta}$, we then use Eq.(9) to iteratively obtain the more accurate reconstructed PCA coefficient vector z. Fig. 3(b) illustrates the comparison results with different solution of optimisation problem on the AR database. As shown in Fig. 3(b), the improved coefficient analytic solution of FSRC-QO consistently achieves better classification results than the previous scheme, regardless of the number of iterations of closed-form solution.

Nevertheless, since $\mathbf{D}_{\mathbf{I}}$ and $\mathbf{U}$ are not in the same subspace, there is a certain flaw in the first phase of the proposed algorithm. In order to overcome this defect, during the label prediction, the discriminatory capacity is exerted 225 on the reconstructed PCA coefficient of each class to map $\mathbf{z}$ and $\mathbf{A}$ to the same subspace when representing a test sample, which can solve the problem of inconsistency of the subspace from the angles of accuracy. Hence we can achieve 
more discriminative face classification results.

\subsection{Computational complexity analysis}

230

All of them are constituted by two steps: 1) calculate the coefficient vector $\boldsymbol{\beta}$ via different approaches; 2) predict test label using $\boldsymbol{\beta}$. Since the former takes up more than $99 \%$ computational time, we would focus on the vector calculation step to discuss the computational complexity. 
Table 1: The computational complexity of proposed methods, where $p$ is the number of instances in dictionary $\mathbf{D}_{\mathbf{I}}, n$ is the number of instances in Training set $\mathbf{A}$, and $d$ is the sample dimension.

\begin{tabular}{c|c}
\hline Method & computational complexity \\
\hline ESRC & $\mathcal{O}\left((n+p)^{3}\right)$ \\
\hline FSRC-QO & $\mathcal{O}\left(p^{2} d+p^{2.373}+p^{3}\right)$ \\
\hline FSRC-QO-CNN & $\mathcal{O}\left(p^{2} d+p^{2.373}+p^{3}\right)$ \\
\hline
\end{tabular}

Furthermore, In the experiment section, we set maxIteration $=30$ in FSRCQO. Since FSRC-QO has solved the "inconsistency of subspace" problem in the second phase, we find even with fewer iterations, FSRC-QO could still achieve good performance.

\section{Experimental results}

\subsection{Experiment setting}

In this section, we demonstrate the efficiency of the proposed two algorithms: FSRC-QO and FSRC-QO-CNN under the undersampled situation on publicly nificant difference based on the assumption that the intraclass variation of any gallery face can be approximated by a sparse linear combination of the intraclass 
differences from sufficient number of generic faces. Hence the variant bases can shows an example of 10 images of one subject from the AR database.

In order to better evaluate our methods under the effect of variations in 


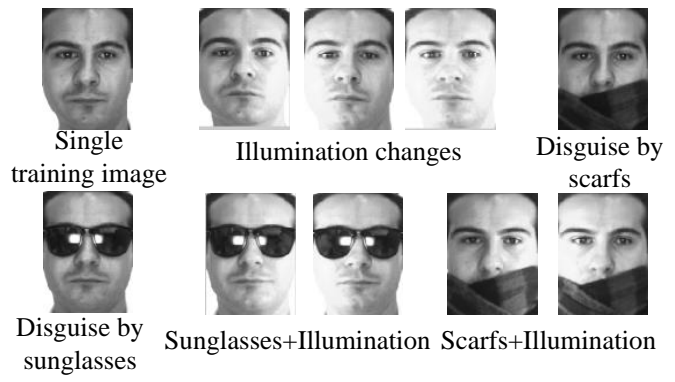

Figure 4: The selected image samples of a subject from AR.

appearance including illumination, disguise and expression in the AR database, we divide the test images from the same subject into six situations: (1) the samples from 2 th to 4 th are selected for illumination changes. (2) the selected 5th sample is disguised by sunglasses. (3) the selected 8th sample is disguised by scarfs. (4) the 6th and 7th samples are represented by the sunglasses with illumination. (5) the 9th and 10th samples are represented by the scarfs and illumination. Table 2 demonstrates the comparison result of each method under the different variations. As shown in Table 2, the improvement achieved by the proposed method using FSRC-QO is much high than those of other traditional methods, the main reason is that the FSRC-QO method enhances the discriminatory capacity using PCA and LDA constrained model in two sequential sparse representation stages, so as to achieve a good sparse representation of a test sample. In summary, the superiority of our algorithm is more obvious.

Meanwhile, the evaluation of execution speed of each method over six variations in appearance is shown in Fig. 5. As can be seen from Fig. 5, the proposed method FSRC-QO shows the promising performance in terms of speed, which 325 is much faster than ESRC [13] across all different variations of data set. It indicates that the optimisation model using dimensionality reduction constraint is more effective for representing the different variations.

To better reveal the influence of the different illuminations conditions for our method, we select a set of experimental results in terms of the reconstruction process of the test sample to achieve a detailed illuminations analysis. Fig. 6 
Table 2: The recognition rates (\%) with the different variations on the AR database

\begin{tabular}{c|c|c|c|c|c}
\hline & \multicolumn{5}{|c}{ Variations } \\
Method & Illumination & $\begin{array}{c}\text { Disguised } \\
\text { by } \\
\text { sunglasses }\end{array}$ & $\begin{array}{c}\text { Disguised } \\
\text { by } \\
\text { scarfs }\end{array}$ & $\begin{array}{c}\text { Sunglasses } \\
+ \\
\text { illumination }\end{array}$ & $\begin{array}{c}\text { Scarfs } \\
+ \\
\text { illumination }\end{array}$ \\
\hline FSRC-QO & $\mathbf{1 0 0 . 0 0}$ & $\mathbf{9 5 . 0 0}$ & $\mathbf{9 2 . 5 0}$ & $\mathbf{9 1 . 2 5}$ & $\mathbf{8 1 . 8 8}$ \\
\hline ESRC [13] & 98.75 & 82.50 & 78.75 & 80.00 & 68.75 \\
\hline SRC [40] & 71.25 & 55.00 & 30.00 & 28.13 & 18.13 \\
\hline CRC [41] & 74.17 & 56.65 & 37.50 & 26.88 & 20.63 \\
\hline LRC [4] & 60.83 & 67.50 & 10.00 & 31.87 & 10.00 \\
\hline CIRLRC [16 & 76.67 & 40.00 & 11.25 & 33.13 & 11.25 \\
\hline TPTSR 43] & 68.33 & 55.00 & 35.00 & 30.63 & 20.00 \\
\hline SRICE [27 & 60.42 & 61.25 & 25.00 & 23.12 & 13.75 \\
\hline
\end{tabular}

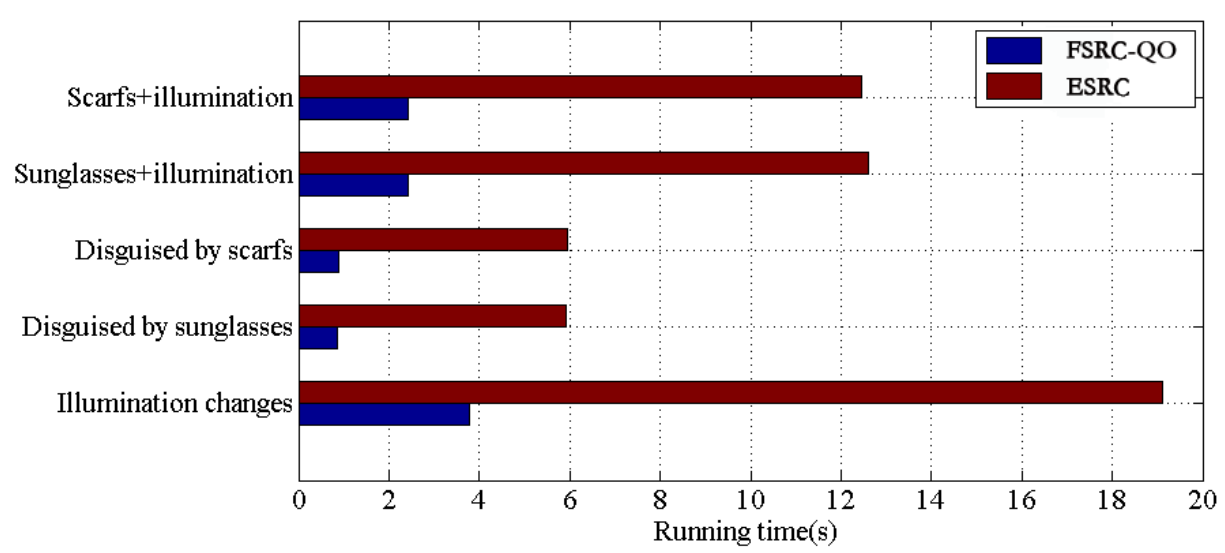

Figure 5: The comparison running time over six variations on the AR database.

shows the reconstruction of $\mathbf{y}$ in the presence of uniform illumination such as (a) and (b) will reduce the weights of samples from within-class, increasing the approximation error of illumination, and potentially leading to misclassification. In contrast, with the nonuniform illumination condition (c), we can effectively reconstruct the illumination information of face images, which lead to correct classification result.

As described in Section 3.1, we project the training samples into the PCA transformed space in which a new sparse representation model with PCA constrained is designed as Eq.(5), in this optimisation process, $\mathbf{U}$ is defined as the 
basis of PCA in terms of training samples. We then make a further analysis for the recostruction process using two reconstructed Eigen faces ordered by the first two principle components of $\mathbf{U}$, as shown in Fig. 7.

Evidently, we can see that the reconstructed first principle component represents the dark information of face images, as well as the second principle component stands for the bright part of faces. It implies that the PCA-based training samples has the capacity to reconstruct the brightness and darkness of the face images, respectively. Nevertheless, from the above experiment, we can see that our method cannot strongly distinguish the illumination from the complexion in the condition of uniform illumination, thus leading to incorrect identification of the class membership of test sample $\mathbf{y}$. We believe this discriminatory power of illumination of our models should be improved in the future work.

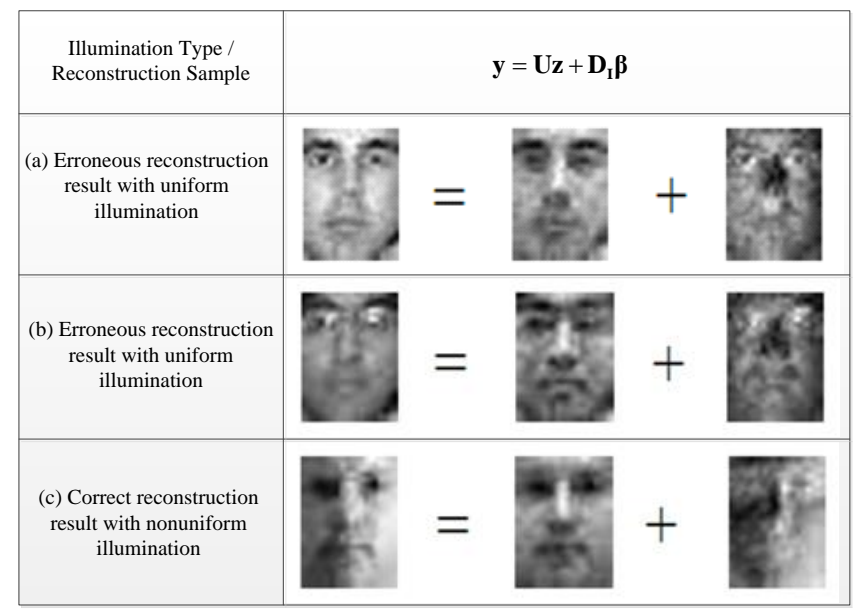

Figure 6: An example of reconstruction of the test sample with different illuminations.

\begin{tabular}{|c|c|}
\hline First principle component & Second principle component \\
\hline & \\
\hline
\end{tabular}

Figure 7: An example of reconstruction of the test sample using two principle components. 
Table 3: Face recognition rates (\%) of differnt state-of-the-art methods on AR

\begin{tabular}{c|c}
\hline Method & Recognition rate (\%) \\
\hline SVM 44 & 96.5 \\
\hline CRC 41 & 98.0 \\
\hline SRC 40 & 97.5 \\
\hline DLSI(C) 45 & 97.5 \\
\hline FDDL 46 & 97.5 \\
\hline LC-KSVD 47 & 97.8 \\
\hline MMDL 48 & 97.3 \\
\hline DPL 49 & 98.3 \\
\hline FSRC-QO-CNN & $\mathbf{9 9 . 7}$
\end{tabular}

Moreover, we compare the proposed FSRC-QO-CNN method with some state-of-the-art methods on AR. The same experiment setting as in [49 is used

to split the original dataset into training and test. Specifically, for each subject, 20 images are selected for training, and the remaining images are used for test. Table 3 demonstrates the different methods such as SVM [44, SRC [40], CRC [4], the state-of-the-art DLSI(C) [4], FDDL 46], LC-KSVD [7], MMDL 48, DPL 49, and the proposed ES-PCA-CNN method. As shown in these tables, the proposed FSRC-QP-CNN method achieve $99.7 \%$ recognition rate, which is better than those of the other state-of-the-art methods.

\subsection{Experiment on FERET}

The FERET face database 36 is a result of the FERET program, which was sponsored by the U.S. Department of Defense through the Defense Advanced

${ }_{65}$ Research Projects Agency program. It has become a benchmark database for the evaluation of face recognition algorithms. The proposed algorithm was evaluated on a subset of FERET, which includes 1400 images of 200 individuals and each subject has seven different images.

In this experiment, the synthesized strategy for intraclass variant dictionary presented in Section 5.1 is performed before classification, by which the first 40 subjects are used to construct the intraclass variant dictionary, the other 160 subjects are employed as the gallery and query set. We resized each face 
image of the FERET database to 40 by 40 . For each subject, a single image is randomly selected for training and the remaining images are used for test. We repeat our experiment 7 times and measured the performance of different face classification algorithms in terms of recognition rate and running time. Some examples of the FERET dataset are shown in Fig. 8

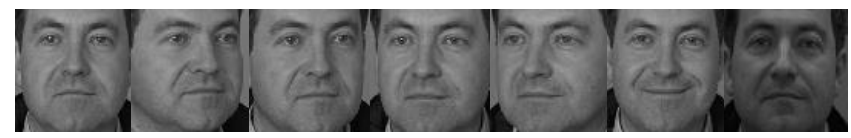

Figure 8: Example faces of the FERET database.

Table 4: The comparison results of different methods on the FERET database

\begin{tabular}{c|c|c}
\hline Method & Recognition rate (\%) & Running time (s) \\
\hline FSRC-QO & 68.96 & 243.75 \\
\hline FSRC-QO-CNN & $\mathbf{9 1 . 7 7}$ & 76.90 \\
\hline ESRC 13 & 58.33 & 2640.51 \\
\hline CIRLRC 16] & 51.08 & 2485.05 \\
\hline TPTSR 43 & 47.83 & 22.76 \\
\hline SRICE 27 & 47.33 & 1424.14 \\
\hline SRC 40] & 38.44 & 541.60 \\
\hline CRC 41 & 37.71 & 124.48 \\
\hline LRC 42 & 43.13 & 24.12 \\
\hline
\end{tabular}

The performance of the proposed FSRC-QO and FSRC-QO-CNN methods are compared to the other typical representation-based classification approaches including ESRC [13, CIRLRC [16, TPTSR [43, SRICE [27, SRC 40], CRC 41] and LRC 42] methods. As shown in Table 4, we can see that the proposed FSRC-QO-CNN achieves the $91.77 \%$ highest recognition rate with running at lower speed, which fully shows a highly competitive advantage over traditional methods in terms of computation complexity and accuracy.

\subsection{Experiment on $L F W$}

Labeled Faces in the Wild (LFW) 37 is one of the most challenging datasets that consists of abundant variations of face images including pose, illumination 
and expression, etc. The database has more than 13000 images of 5749 individuals with different gender, ages, etc. The proposed algorithms are evaluated on a subset of LFW database, which includes 1580 images of 158 individuals with 10 different images of each subject. We resize each LFW face image to 64 by 64. In this experiment, the first 58 subjects are used to construct the intraclass variant dictionary which contains 522 bases ( 9 bases for each generic subject), and the other 100 subjects are employed as the gallery and query set. For each subject, a single image is randomly selected for training and the remaining images are used for test. We repeat our experiment 10 times and measured the performance of different face classification algorithms in terms of recognition rate and running time. Some images from the LFW database are shown in Fig. 9

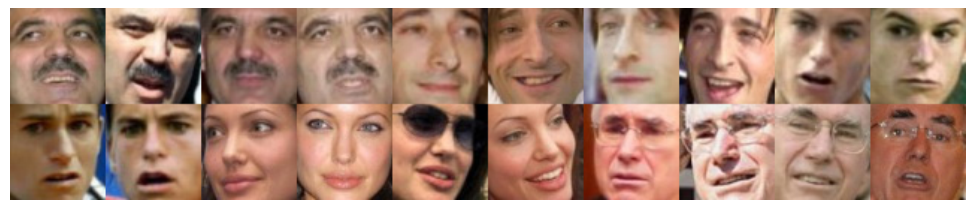

Figure 9: Example faces of the LFW database.

Table 5: The comparison results of different methods on the LFW database

\begin{tabular}{c|c|c}
\hline Method & Recognition rate (\%) & Running time (s) \\
\hline FSRC-QO & 23.67 & 325.75 \\
\hline FSRC-QO-CNN & $\mathbf{6 1 . 5 6}$ & 143.95 \\
\hline ESRC [1] & 12.56 & 1761.47 \\
\hline CIRLRC [16 & 12.22 & 1399.26 \\
\hline TPTSR 43 & 9.78 & 28.30 \\
\hline SRICE [27 & 9.78 & 919.00 \\
\hline SRC 40] & 8.56 & 349.89 \\
\hline CRC 41 & 8.67 & 7.52 \\
\hline LRC 42 & 5.44 & 12.32 \\
\hline
\end{tabular}
compared to the other typical representation-based classification approaches including ESRC [13, CIRLRC [16], TPTSR [43], SRICE [27, SRC [40, CRC [1] 
and LRC 42 methods. In light of the experimental results as shown in Table 5 we can see that the proposed FSRC-QO, FSRC-QO-CNN methods respectively achieve $23.67 \%$ and $61.56 \%$ recognition rate, which outperform the other traditional methods in terms of accuracy. Compared to the classical ESRC method, it should be noted that our FSRC-QO-CNN can achieve highly competitive performance in computational cost.

\subsection{Experiment on FRGC}

For the face recognition grand challenge (FRGC) version 2 database, we use a similar split as that on the LFW database to construct the training and test sets. The FRGC database [38] consists of controlled and uncontrolled color face images. The controlled images show good image quality, while the uncontrolled ones with poor image quality are taken under the complex backgrounds. In this experiment, we select 100 individuals with 30 different images of each subject from FRGC to construct the experimental subset, and we resize each face image in FRGC to 80 by 80 . The intraclass variant dictionary is constructed from the first 20 subjects that contains 580 bases (29 bases for each generic subject), the remaining 80 subjects are employed as the gallery and query set. In each subject, a single image is randomly selected for training and the remaining 29 images are used for test. We repeat our experiment 10 times and measured the performance of different face classification algorithms in terms of recognition rate and running time. Some images from the FRGC database are shown in Fig. 10.

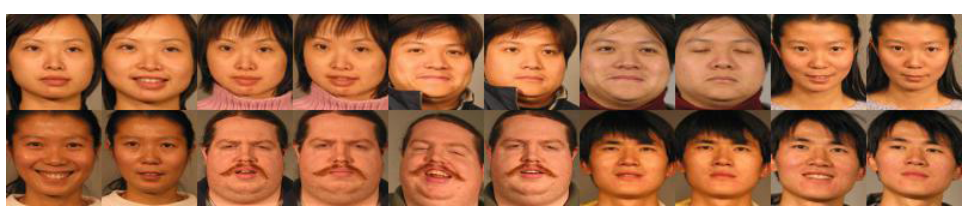

Figure 10: Example faces of the FRGC database.

Table 6 presents the recognition rates achieved by a set of traditional methods including ESRC [13, CIRLRC [16], TPTSR [43, SRICE [27, SRC [40, 
CRC [4] and LRC [42] methods. The proposed FSRC-QP-CNN method achieves the $97.72 \%$ highest recognition rate with running at lower speed, which once again shows a highly competitive advantage over traditional methods in terms of computational complexity and accuracy.

\begin{tabular}{|c|c|c|}
\hline Method & Recognition rate (\%) & Running time (s) \\
\hline FSRC-QO & 50.18 & 2696.54 \\
\hline FSRC-QO-CNN & 97.72 & 412.64 \\
\hline ESRC 13 & 23.71 & 18553.25 \\
\hline CIRLRC 16 & 22.54 & 3223.16 \\
\hline TPTSR 43 & 20.65 & 216.05 \\
\hline SRICE 27 & 20.71 & 2317.82 \\
\hline SRC 40] & 23.06 & 2396.06 \\
\hline CRC 41 & 23.19 & 58.17 \\
\hline LRC 42 & 16.16 & 73.23 \\
\hline
\end{tabular}

From the above experiment results, it should be noted that the improvements achieved by the proposed method on the LFW and FRGC datasets are much higher than those on the other datasets. This is mainly because the LFW and FRGC datasets contain more variations in appearance than the other datasets.

435 In such scenarios, the superiority of our algorithm is more evident compared to other sparse (or collaborative) representation-based methods.

\subsection{Experiment comparisons with some deep learning based methods}

In this section, we compare the proposed FSRC-QO-NN method with some state-of-the-art deep models such as NN-CNN [50], VGG-SVM [51] and VGG3DPD-CRC [52]. The experiments were conducted on some well-known public face datasets, including AR, LFW and FRGC. Note that all the methods of this experiment are based on the well trained deep learning model. More specifically, we first apply the VGG-FACE model to all the training and test images for robust facial features extraction, using computation of the forward propagation 445 to obtain the convolution features. We then perform the face classification 
using NN classifier with Euclidean distance, SVM, 3DPD-CRC and the proposed FSRC-QO method, respectively. The face recognition rates are reported in Table. 7. As seen in Table. 7, the proposed FSRC-QO-CNN method achieves better result than other methods in terms of recognition rate. This is attributed to the fact that the more robust sparse representation is performed based on the proposed quadratic optimisation processing. Furthermore, it should be noted that FSRC-QO-CNN is based on the well trained feature maps obtained by our first strategy, and the training processing can be completed using offline mode. Hence the dimensionality of raw face images are reduced by the DLbased training model, which can be regarded as a data preprocessing stage.

\subsection{Experiment analysis}

In this section, to show clearly how much contribution of each of the two strategies makes, we conduct two verified experiments on the ORL and FERET datasets. More specifically, the first strategy of the proposed method can be divided into two steps by which the quadratic optimisation is performed in downsized coefficient solution subspace of PCA and LDA, respectively. We named this two steps as "ES-PCA" and "ES-LDA". As show in Table. 8 and Table. 9, the designed two steps of the first strategy of our method are built on the foundation of the traditional ESRC. Meanwhile, the second strategy of our method is actually an enhancement of the first one and it is intended to improve the accuracy, instead of to speed up ESRC. In light of the experimental results achieved by the different phases mentioned above, we can conclude that the proposed method achieves more effective and stable performance both in recognition rate and execution speed.

Furthermore, from the experimental results reported in different datasets, it should be noted that the improvements achieved by our method on the FRGC and LFW databases are much higher than those achieved on the other datasets. The main reason is that the FRGC and LFW datasets contain more variations 
scenarios, the superiority of our method is more evident compared with other sparse (or collaborative) representation-based methods.

Table 7: Comparison results of DL-based approaches on different datasets

\begin{tabular}{c|c|c|c|c}
\hline Dataset & NN-CNN & VGG-SVM & VGG-3DPD-CRC & FSRC-QO-CNN \\
\hline AR & 97.29 & 97.63 & 98.86 & $\mathbf{9 9 . 7 0}$ \\
\hline LFW & 54.11 & 54.92 & 60.24 & $\mathbf{6 1 . 5 6}$ \\
\hline FRGC & 91.43 & 91.84 & 93.92 & $\mathbf{9 7 . 7 2}$ \\
\hline
\end{tabular}

Table 8: Comparison result of each optimisation phase on ORL

\begin{tabular}{c|c|c}
\hline Methon & Recognition rate (\%) & Running time (s) \\
\hline ESRC & 63.28 & 264.55 \\
\hline ES-PCA & 78.13 & 90.24 \\
\hline ES-LDA & 82.03 & 37.50 \\
\hline FSRC-QO-CNN & $\mathbf{9 8 . 4 5}$ & $\mathbf{2 2 . 7 3}$ \\
\hline
\end{tabular}

Table 9: Comparison result of each optimisation phase on FERET

\begin{tabular}{c|c|c}
\hline Methon & Recognition rate (\%) & Running time (s) \\
\hline ESRC & 58.33 & 2640.51 \\
\hline ES-PCA & 61.46 & 405.04 \\
\hline ES-LDA & 68.96 & 243.75 \\
\hline FSRC-QO-CNN & $\mathbf{9 1 . 7 7}$ & $\mathbf{7 6 . 9 0}$ \\
\hline
\end{tabular}

\section{Conclusion}

In this paper, we proposed two fast SRC algorithms for face classification.

The key innovation of the proposed work is to accomplish face recognition using a novel dimensionality reduction optimisation model, which makes SRC robust to the different variations in appearance. The strength of the technique lies in successfully constructing a quadratic optimisation in downsized coefficient solution subspace, and thereby enhancing the discriminatory capacity of the dictionary to reconstruct input signals faithfully. We believe that our promising 
results can encourage more work on synthesizing more informative optimisation structure and increase this paper for better SRC solutions.

\section{Acknowledgement}

This work was partially supported by the National Key Research and Development Program of China (Grant No. 2017YFC1601800, 2016YFD0401204), the Engineering and Physical Sciences Research Council Programme Grants FACER2VM (EP/N007743/1), the National Natural Science Foundation of China (Grant No. 61876072, 61672263, 61702226), the Natural Science Foundation of Jiangsu Province (Grant No. BK20161135), China Postdoctoral Science

Foundation (Grant no. 2018T110441), Six Talent Peaks Project in Jiangsu Province (Grant No. XYDXX-012) and the 111 Project (Grant No. B12018).

\section{References}

[1] P. Koppen, Z.-H. Feng, J. Kittler, M. Awais, W. Christmas, X.-J. Wu, H.-F. Yin, Gaussian mixture 3d morphable face model, Pattern Recognition.

[2] R. Senthilkumar, R. K. Gnanamurthy, A comparative study of $2 \mathrm{~d}$ pca face recognition method with other statistically based face recognition methods, Journal of The Institution of Engineers (India): Series B 97 (3) (2016) 1-6.

[3] J. Lu, K. N. Plataniotis, A. N. Venetsanopoulos, Face recognition using lda-based algorithms, IEEE Transactions on Neural Networks 14 (1) (2003) 195-200.

[4] M. Belkin, P. Niyogi, Laplacian eigenmaps for dimensionality reduction and data representation, Neural Computation 15 (6) (2003) 1373-1396.

[5] Y. Yang, Y. Wang, X. Xue, Discriminant sparse locality preserving projection for face recognition, Multimedia Tools and Applications 76 (2) (2017) 2697-2712. 
[6] S. T. Roweis, L. K. Saul, Nonlinear dimensionality reduction by locally linear embedding, Science 290 (5500) (2000) 2323-2326.

[7] Y. Xu, Q. Zhu, Z. Fan, Y. Wang, J. S. Pan, From the idea of "sparse representation" to a representation-based transformation method for feature extraction, Neurocomputing 113 (2013) 168-176.

[8] X. Ma, W. Zhuang, Y. Li, J. Feng, Sparse representation based undersampled face recognition with shared prototypeauxiliary dictionaries, Neurocomputing 239 (2017) 58-68.

[9] W. Yang, Z. Wang, C. Sun, A collaborative representation based projections method for feature extraction, Pattern Recognition 48 (1) (2015) $20-27$.

[10] Y. Su, X. Gao, X.-C. Yin, Fast alignment for sparse representation based face recognition, Pattern Recognition 68 (2017) 211-221.

[11] Y. Chen, J. Su, Sparse embedded dictionary learning on face recognition, Pattern Recognition 64 (2017) 51-59.

[12] L. F. Chen, H. Y. M. Liao, M. T. Ko, J. C. Lin, G. J. Yu, A new lda-based face recognition system which can solve the small sample size problem, Pattern Recognition 33 (10) (2000) 1713-1726.

[13] W. Deng, J. Hu, J. Guo, Extended src: undersampled face recognition via intraclass variant dictionary, IEEE Transactions on Pattern Analysis and Machine Intelligence 34 (9) (2012) 1864-1870.

[14] M. Yang, D. Zhang, D. Zhang, S. Wang, Relaxed collaborative representation for pattern classification, in: IEEE Conference on Computer Vision and Pattern Recognition, Vol. 157, 2012, pp. 2224-2231.

${ }_{535}$ [15] P. Zhu, W. Zuo, L. Zhang, C. K. Shiu, Image set-based collaborative representation for face recognition, IEEE Transactions on Information Forensics and Security 9 (7) (2013) 1120-1132. 
[16] Y. Xu, X. Li, J. Yang, Z. Lai, D. Zhang, Integrating conventional and inverse representation for face recognition., IEEE Transactions on Cybernetics 44 (10) (2014) 1738-1746.

[17] X. Song, Z. H. Feng, G. Hu, X. J. Wu, Half-face dictionary integration for representation-based classification, IEEE Transactions on Cybernetics 47 (1) (2017) 142-152.

[18] S. Liu, X. Zhang, Y. Peng, H. Cao, Virtual images inspired consolidate collaborative representation-based classification method for face recognition, Journal of Modern Optics 63 (12) (2016) 1-8.

[19] Y. Du, Y. Wang, Generating virtual training samples for sparse representation of face images and face recognition, Journal of Modern Optics 63 (6) (2016) 1-9.

[20] X. Song, Z.-H. Feng, G. Hu, J. Kittler, W. Christmas, X.-J. Wu, Dictionary integration using $3 \mathrm{~d}$ morphable face models for poseinvariant collaborative-representation-based classification, arXiv preprint arXiv:1611.00284.

[21] C. Shao, X. Song, Z.-H. Feng, X.-J. Wu, Y. Zheng, Dynamic dictionary optimization for sparse-representation-based face classification using local difference images, Information Sciences 393 (2017) 1-14.

[22] Z. H. Feng, G. Hu, J. Kittler, W. Christmas, X. J. Wu, Cascaded collaborative regression for robust facial landmark detection trained using a mixture of synthetic and real images with dynamic weighting., IEEE Transactions on Image Processing 24 (11) (2015) 3425-3440.

[23] S. Liu, Y. Zhang, X. Yang, D. Shi, J. J. Zhang, Robust facial landmark detection and tracking across poses and expressions for in-the-wild monocular video, Computational Visual Media 3 (1) (2017) 1-15.

[24] Z. H. Feng, J. Kittler, W. Christmas, P. Huber, X. J. Wu, Dynamic attention-controlled cascaded shape regression exploiting training data aug- 
mentation and fuzzy-set sample weighting, in: IEEE Conference on Computer Vision and Pattern Recognition(CVPR), 2017.

[25] Z.-H. Feng, J. Kittler, W. Christmas, X.-J. Wu, A unified tensor-based active appearance face model, arXiv preprint, arXiv:1612.09548.

[26] J. Kittler, P. Huber, Z.-H. Feng, G. Hu, W. Christmas, 3d morphable face models and their applications, in: International Conference on Articulated Motion and Deformable Objects, Springer, 2016, pp. 185-206.

[27] X. Song, Z. Liu, X. Yang, S. Gao, A new sparse representation-based classification algorithm using iterative class elimination, Neural Computing and Applications 24 (7) (2014) 1627-1637.

[28] Z. H. Feng, P. Huber, J. Kittler, W. Christmas, Random cascadedregression copse for robust facial landmark detection, IEEE Signal Processing Letters 22 (1) (2015) 76-80.

[29] Z.-H. Feng, J. Kittler, M. Awais, P. Huber, X.-J. Wu, Face detection, bounding box aggregation and pose estimation for robust facial landmark localisation in the wild, in: The IEEE Conference on Computer Vision and Pattern Recognition (CVPR) Workshops, 2017, pp. 160-169.

[30] Z. Zhou, Y. Wang, Q. M. J. Wu, C. N. Yang, X. Sun, Effective and efficient global context verification for image copy detection, IEEE Transactions on Information Forensics and Security 12 (1) (2017) 48-63.

[31] Z. Xia, X. Wang, L. Zhang, Z. Qin, X. Sun, K. Ren, A privacy-preserving and copy-deterrence content-based image retrieval scheme in cloud computing, IEEE Transactions on Information Forensics and Security 11 (11) (2017) 2594-2608.

[32] J. Li, X. Li, B. Yang, X. Sun, Segmentation-based image copy-move forgery detection scheme, IEEE Transactions on Information Forensics and Security 10 (3) (2017) 507-518. 
[33] O. M. Parkhi, A. Vedaldi, A. Zisserman, Deep face recognition, in: Proceedings of the British Machine Vision Conference (BMVC), 2015, pp. 41.141.12 .

[34] A. Y. Yang, Z. Zhou, A. G. Balasubramanian, S. S. Sastry, Y. Ma, Fast $\ell_{1}$ Minimization Algorithms for Robust Face Recognition, IEEE Transactions on Image Processing 22 (8) (2013) 3234-3246.

[35] http://cobweb.ecn.purdue.edu/aleix/aleix-face-DB.html.

[36] http://www.itl.nist.gov/iad/humanid/feret/feret-master.html.

[37] G. B.Huang, M. Mattar, T. Berg, E. Learned-Miller, Labeled faces in the wild: A database forstudying face recognition in unconstrained environments, 2008.

[38] P. J. Phillips, P. J. Flynn, T. Scruggs, K. W. Bowyer, J. Chang, K. Hoffman, J. Marques, J. Min, W. Worek, Overview of the face recognition grand challenge, in: IEEE Computer Society Conference on Computer Vision and Pattern Recognition, 2005, pp. 947-954.

[39] D. M. Malioutov, M. Cetin, A. S. Willsky, Homotopy continuation for sparse signal representation, in: IEEE International Conference on Acoustics, Speech, and Signal Processing, Vol. 5, 2005, pp. 733-736.

[40] B. C. Krishna, V. H. Bindu, K. L. Durga, G. Lokeshwar, G. Ashokkumar, An efficient face recognition system by declining rejection rate using pca, International Journal of Engineering Science and Advanced Technology 2 (1) (2012) 93-98.

[41] L. Zhang, M. Yang, X. Feng, Sparse representation or collaborative representation: Which helps face recognition?, in: IEEE International Conference on Computer Vision, 2012, pp. 471-478.

[42] I. Naseem, R. Togneri, M. Bennamoun, Linear regression for face recognition, IEEE Transactions on Pattern Analysis and Machine Intelligence 32 (11) (2010) 2106-2112. 
[43] Y. Xu, D. Zhang, J. Yang, J. Y. Yang, A two-phase test sample sparse representation method for use with face recognition, IEEE Transactions on Circuits and Systems for Video Technology 21 (9) (2011) 1255-1262.

[44] J. A. Suykens, J. Vandewalle, Least squares support vector machine classifiers, Neural processing letters 9 (3) (1999) 293-300.

[45] I. Ramirez, P. Sprechmann, G. Sapiro, Classification and clustering via dictionary learning with structured incoherence and shared features, in: Computer Vision and Pattern Recognition (CVPR), 2010 IEEE Conference on, IEEE, 2010, pp. 3501-3508.

[46] M. Yang, L. Zhang, X. Feng, D. Zhang, Fisher discrimination dictionary learning for sparse representation, in: Computer Vision (ICCV), 2011 IEEE International Conference on, IEEE, 2011, pp. 543-550.

[47] Z. Jiang, Z. Lin, L. S. Davis, Label consistent k-svd: Learning a discriminative dictionary for recognition, IEEE Transactions on Pattern Analysis and Machine Intelligence 35 (11) (2013) 2651-2664.

[48] Z. Wang, J. Yang, N. Nasrabadi, T. Huang, A max-margin perspective on sparse representation-based classification, in: Proceedings of the IEEE International Conference on Computer Vision, 2013, pp. 1217-1224.

[49] S. Gu, L. Zhang, W. Zuo, X. Feng, Projective dictionary pair learning for pattern classification, in: Advances in Neural Information Processing Systems, 2014, pp. 793-801.

[50] S.-H. Wu, Y.-W. Zhong, J. Yao, 2, 3-di (2-pyridyl)-5-phenylpyrazine: A nn-cnn-type bridging ligand for dinuclear transition-metal complexes, Chemistry-An Asian Journal 8 (7) (2013) 1504-1513.

${ }_{645}$ [51] A. Vijayalakshmi, et al., Deep learning approach to detect malaria from microscopic images, Multimedia Tools and Applications 1-21. 
[52] X. Song, Z.-H. Feng, G. Hu, J. Kittler, X.-J. Wu, Dictionary integration using $3 \mathrm{~d}$ morphable face models for pose-invariant collaborativerepresentation-based classification, IEEE Transactions on Information Forensics and Security 13 (11) (2018) 2734-2745. 